\section{A Sequence Resource of Autosomes and Additional Chromosomes in the Peach Pathotype of Alternaria alternata}

\author{
Koichiro Ushijima and Mikihiro Yamamoto ${ }^{\dagger}$ \\ Graduate School of Environmental and Life Science, Okayama University, Okayama 700-8530, Japan
}

\begin{abstract}
Alternaria alternata is a generally saprophytic fungus. Its genome consists of 10 autosomes, while some strains have one or two additional chromosomes, called a conditionally dispensable chromosome (CDC). A CDC is not required for reproduction but confers hostspecific pathogenicity. We sequenced the genome of the peach pathotype of $A$. alternata using Nanopore and assembled it into 20 sequences. The 10 largest sequences corresponded to 10 gapless sequences of $A$. solani autosomes, and 1 sequence was a mitochondrial genome. Nine other sequences may be derived from CDCs because of lack of similarity with autosomes of other Alternaria spp. The sequence information could provide novel insights into genomes of Alternaria spp. and on the biosynthesis of a novel host-specific toxin in the peach pathotype of $A$. alternata.
\end{abstract}

\section{Genome Announcement}

Alternaria alternata, one of the small-spored Alternaria spp., is generally a ubiquitous saprophytic fungus frequently found on decaying plant tissues. However, some strains exhibit host-specific pathogenicity to plants. Eight pathotypes (Japanese pear, strawberry, tangerine, apple, tomato, tobacco, rough lemon, and peach) have been reported and their hostspecific toxins (HSTs) produced, which exhibited high levels of pathogenicity to their host plants (Iwamoto et al. in press; Tsuge et al. 2016). The autosome genes did not confer the pathogenicity and the toxins were biosynthesized by genes located on conditionally dispensable chromosomes (CDCs). CDCs are not typically required for saprophytic growth and reproduction on artificial media, whereas a range of genes related to HST biosynthesis are clustered on CDC, except for the peach pathotype. The peach pathotype causing black spots on peach was first isolated from lesions of leaves, twigs, and fruit of peach cultivar Shimizu Hakuto orchards in Okayama Prefecture, Japan (Inoue and Nasu 2000). Although the disease was apparent only in Shimizu Hakuto orchards, other peach cultivars were found to be somewhat susceptible - but not immune - to spore inoculation at a higher density and sensitive to HST (AP-toxin) at a higher concentration in laboratory bioassays. Host plants of other pathotypes showed no response to severe bioassays (Iwamoto et al. in press). Draft genome sequences of multiple species of Alternaria have been released. The genomes were highly fragmented because they were assembled from short reads sequenced by secondgeneration sequencers (Dang et al. 2015). Recently, four long, continuous-draft genomes were reported, which were assembled from long reads sequenced using the third-generation

\footnotetext{
${ }^{\dagger}$ Corresponding author: M. Yamamoto; myama@okayama-u.ac.jp
}

The author(s) declare no conflict of interest.

Accepted for publication 23 May 2019.
Keywords

genomics, metabolomics, proteomics 
Table 1. Overview of draft genome sequence of Alternaria alternata pathotype peach

\begin{tabular}{|c|c|c|c|c|c|c|c|c|c|c|}
\hline \multirow[b]{2}{*}{ Name } & \multirow[b]{2}{*}{ Length (bp) } & \multirow[b]{2}{*}{ Genes $^{c}$} & \multirow[b]{2}{*}{ Ends ${ }^{d}$} & \multicolumn{3}{|c|}{ BUSCO analysis $^{a}$} & \multicolumn{4}{|c|}{ Similarity to other genomes ${ }^{b}$} \\
\hline & & & & Com & Dup & Frg & A. solani & A. alternata & A. tenuissima & A. gaisen \\
\hline Ch01 & $6,819,131$ & 2,452 & Both $^{e}$ & 55 & 0 & 0 & Ch1 & 1,5 & $2,6,8$ & 1,2 \\
\hline Ch02 & $5,908,156$ & 2,114 & Both & 39 & 0 & 0 & Ch2 & 12,2 & $1,12,17$ & 9,2 \\
\hline $\mathrm{ChO3}$ & $3,326,390$ & 1,174 & Both & 28 & 0 & 0 & Ch3 & 3 & $3,16 ?$ & 3 \\
\hline Ch04 & $3,070,597$ & 1,098 & Both & 23 & 0 & 0 & Ch4 & 13,6 & 5 & 4 \\
\hline Ch05 & $2,800,883$ & 999 & Both & 40 & 1 & 0 & Ch5 & 4 & 4 & 6,13 \\
\hline Ch06 & $2,607,085$ & 896 & Both & 13 & 0 & 0 & Ch6 & 8 & $11,13 ?$ & $7,15 ?$ \\
\hline Ch07 & $2,623,421$ & 876 & $5^{\prime}$ & 30 & 0 & 1 & Ch7 & 7 & 7 & 10,11 \\
\hline Ch08 & $2,399,115$ & 805 & Both $^{e}$ & 19 & 0 & 0 & Ch8 & 9 & 10 & 8 \\
\hline Ch09 & $2,433,500$ & 872 & Both & 21 & 0 & 0 & Ch9 & 10 & 9 & 5 \\
\hline Ch10 & $1,821,462$ & 618 & Both $^{\mathrm{e}}$ & 18 & 0 & 0 & Ch10 & 11 & 6 & 2 \\
\hline Subtotal & $33,809,740$ & 11,904 & & 286 & 1 & 1 & $\ldots$ & $\ldots$ & & $\ldots$ \\
\hline Scaffold01 & 664,702 & 175 & $3^{\prime}$ & 0 & 0 & 0 & $\ldots$ & $\ldots$ & 14,19 & $\ldots$ \\
\hline Scaffold02 & 202,251 & 61 & No & 0 & 1 & 0 & $\ldots$ & $\ldots$ & $\ldots$ & $\ldots$ \\
\hline Scaffold03 & 755,045 & 227 & $3^{\prime e}$ & 0 & 0 & 0 & $\ldots$ & $\ldots$ & 15 & 12 \\
\hline Scaffold04 & 82,251 & 17 & No & 0 & 0 & 0 & $\ldots$ & $\ldots$ & $\ldots$ & $\ldots$ \\
\hline Scaffold05 & 51,038 & 18 & No & 0 & 0 & 0 & $\ldots$ & $\ldots$ & $\ldots$ & $\ldots$ \\
\hline Scaffold06 & 51,817 & 18 & No & 0 & 0 & 0 & $\ldots$ & $\ldots$ & $\ldots$ & $\ldots$ \\
\hline Scaffold07 & 55,744 & 16 & No & 0 & 0 & 0 & $\ldots$ & $\ldots$ & $\ldots$ & $\ldots$ \\
\hline Scaffold08 & 45,891 & 16 & No & 0 & 0 & 0 & $\ldots$ & $\ldots$ & $\ldots$ & $\ldots$ \\
\hline Scaffold09 & 121,527 & 40 & $3^{\prime}$ & 0 & 0 & 0 & $\ldots$ & $\ldots$ & 18 & $\ldots$ \\
\hline Subtotal & $2,030,266$ & 588 & $\ldots$ & 0 & 1 & 0 & $\ldots$ & $\ldots$ & $\ldots$ & $\ldots$ \\
\hline Total & $35,840,006$ & 12,492 & $\ldots$ & $\ldots$ & $\ldots$ & $\ldots$ & $\ldots$ & $\ldots$ & $\ldots$ & $\ldots$ \\
\hline
\end{tabular}

a Com = complete, Dup = duplicated, and Frg = fragmented.

b Chromosome, scaffold, or contig number of $A$. solani (Wolters et al. 2018), A. alternata ATCC 24957 (Nguyen et al. 2016), A. tenuissima FERA 116, and A. gaisen FERA 650 (Armitage et al. 2019).

c Genes were predicted by augustus.

d Telomere ends.

e The $3^{\prime}$ end was manually extended after assembly.

sequencers PacBio RSII or Nanopore (Armitage et al. 2019; Nguyen et al. 2016; Wolters et al. 2018). Wolters et al. (2018) sequenced $A$. solani and provided gapless sequences for 10 autosomes, although this species seems to have no CDCs. In the present study, we sequenced the chromosomes of $A$. alternata pathotype peach (Iwamoto et al. in press) using Nanopore and generated almost complete autosome sequences and long continuous sequences.

We first estimated the genome size of $A$. alternata pathotype peach, based on k-mer frequency. Six DNA-seq libraries from three pathogenic strains were constructed and sequenced using Illumina HiSeq 2500. In total, $3.5 \mathrm{Gbp}$ of 27 million reads were obtained and k-mer frequency analysis using Jellyfish 2.2.7 software (Marçais and Kingsford 2011) estimated the genome size as approximately $36.6 \mathrm{Mbp} / \mathrm{C}$, which is similar to those of other Alternaria genomes (Woudenberg et al. 2015).

Genomic DNA of a strain was further sequenced using a Flow Cell (R9.4) on a MinION device and MinKNOW 2 (Oxford Nanopore Technologies) according to the manufacturer's manual "1D gDNA long reads without BluePippin," and the base calling was conducted using albacore 2.3 (Oxford Nanopore Technologies) after sequencing. The contaminated adapter sequences were trimmed from the passed sequences using PoreChop (https://github.com/ rrwick/Porechop). Finally, 2.9 Gbp of 0.3 million reads (average $9.6 \mathrm{kbp}$ ) were obtained. Canu 1.7, a de novo assembly software (Koren et al. 2017), was used to assemble the reads and generate 21 contigs. Whole-genome comparison using mummer 3.0 (Kurtz et al. 2004) revealed that the 10 longest contigs corresponded to 10 autosome sequences of $A$. solani (Wolters et al. 2018). Based on chromosome number in the $A$. solani genome, the 10 contigs were designated as Ch01 to Ch10. To assesses quality of assembly, we searched tandem repeat sequences at the $5^{\prime}$ and $3^{\prime}$ ends using Tandem Repeats Finder 4.09 (Benson 1999) and found 16 telomeric regions containing "TTAGGG" repeats, which were similar to telomere sequences of $A$. solani, from 10 autosome contigs. Four chromosomes (Ch01, Ch07, Ch08, and $\mathrm{Ch} 10$ ) lacked telomere sequences at the $3^{\prime}$ end. Whole-genome comparison revealed that the contigs were unlikely to lack the large sequences at terminal regions and it was expected that the regions lacking large sequences where telomere sequences were contained, were short. We attempted to extend the $3^{\prime}$ region of four chromosomes manually. Corrected reads, which Canu generated during the assembly process, were mapped on 
contig sequences using minimap2 ( $\mathrm{Li}$ 2018) and the reads mapped on the $3^{\prime}$ ends of the contigs lacking telomere sequences were isolated. Alignments were generated using CLC Genomic Workbench (CLC Bio) from the isolated reads and the consensus sequences of the $3^{\prime}$ end of Ch01, Ch08, and Ch10 were obtained, in which telomere sequences were found at the 3 ' ends. For Ch07, the manual end extension failed; however, a $61 \mathrm{kbp}$ contig, one of 11 remaining contigs, was scaffolded to the $3^{\prime}$ end of Ch07 using SSPACE-LongRead software (Boetzer and Pirovano 2014) and the Nanopore raw reads. The scaffolded 61-kbp contig did not contain telomere sequences and comprised long tandem repeated sequences, which prevented manual sequence extension. We further designated one of the remaining contigs as a mitochondrial genome. The contig sequence was similar to the mitochondrial genome of Alternaria (Liao et al. 2017) and Fusarium (Pantou et al. 2008), and the Canu assembler indicated that the contig structure was circular, although other contigs were predicted to have linear structures. The remaining 9 sequences, designated as scaffold01 to -09 , were speculated to be derived from CDCs because no sequences that were similar to scaffold01 to -09 were found in the draft genomes of $A$. solani and $A$. alternata that had no CDCs. Indeed, a homology search using mummer showed that some scaffolds were partially similar to those of the contigs predicted as CDCs in $A$. tenuissima (Table 1).

Sequences assembled from reads sequenced by third-generation sequencers contain many errors and require polishing using Illumina reads. Our initial sequences also had many errors, because BUSCO analysis demonstrated that only $70 \%$ of fungus core genes were complete. To polish assembled sequences, Illumina DNA-seq reads were mapped to the assembled genomic sequences and consensus sequences were extracted using CLC Genomic Workbench. We provided 19 genomic sequences with $35.8 \mathrm{Mb}$ (Table 1), which corresponded to the genome sizes estimated using k-mer frequency analysis. BUSCO analysis demonstrated that $98.9 \%$ of the fungi dataset was contained in the polished sequences and almost all core genes were located at the autosomes (Ch01 to Ch10). Only one core gene was found at scaffold02 but the gene was duplicated and another was located at Ch05. Gene prediction using augustus 2.3 (Stanke et al. 2006) predicted 11,904 and 588 genes at autosomes and scaffolds, respectively. Previous reports estimated that 209 and 512 genes were located at CDCs of $A$. arborescens pathotype tomato ( $\mathrm{Hu}$ et al. 2012) and A. alternata pathotype tangerine (Wang et al. 2017), respectively. If the scaffolds were derived from CDCs, 588 genes were predicted at the scaffolds in the present study, which may be a reasonable number, and most genes of CDC of $A$. alternata pathotype peach were expected to be involved in our assembly. This suggested that our draft genome would offer novel insights into the regulation of biosynthesis of host-selective toxins.

Nanopore and Illumina read data reported are available in the DDBJ Sequenced Read Archive under the accession numbers DRA008134 and DRA008349, respectively. The accession numbers for sequences assembled in this study are BJEP01000001 to BJEP01000019.

\section{Acknowledgments}

Computations were partially performed on the NIG supercomputer at ROIS National Institute of Genetics.

\section{Literature Cited}

Armitage, A. D., Cockerton, H. M., Sreenivasaprasad, S., Woodhall, J., Lane, C., Harrison, R. J., and Clarkson, J. P. 2019. Genomics, evolutionary history and diagnostics of the Alternaria alternata species group including apple and Asian pear pathotypes. bioRxiv Article 534685 .

Benson, G. 1999. Tandem repeats finder: A program to analyze DNA sequences. Nucleic Acids Res. 27:573-580.

Boetzer, M., and Pirovano, W. 2014. SSPACE-LongRead: Scaffolding bacterial draft genomes using long read sequence information. BMC Bioinf. 15:211.

Dang, H. X., Pryor, B., Peever, T., and Lawrence, C. B. 2015. The Alternaria genomes database: A comprehensive resource for a fungal genus comprised of saprophytes, plant pathogens, and allergenic species. BMC Genomics 16:239.
Hu, J., Chen, C., Peever, T., Dang, H., Lawrence, C., and Mitchell, T. 2012. Genomic characterization of the conditionally dispensable chromosome in Alternaria arborescens provides evidence for horizontal gene transfer. BMC Genomics 13: 171.

Inoue, K., and Nasu, H. 2000. Black spot of peach caused by Alternaria alternata (Fr.) Keissler. J. Gen. Plant Pathol. 66:18-22.

Iwamoto, K., Takamatsu, S., and Yamamoto, M. A host-specific toxin produced by the causal pathogen of black spot of peach. J. Gen. Plant Pathol. In press. doi: 10.1007/s10327-019-00859-5

Koren, S., Walenz, B. P., Berlin, K., Miller, J. R., Bergman, N. H., and Phillippy, A. M. 2017. Canu: Scalable and accurate long-read assembly via adaptive $k$-mer weighting and repeat separation. Genome Res. 27:722-736. 
Kurtz, S., Phillippy, A., Delcher, A. L., Smoot, M., Shumway, M., Antonescu, C., and Salzberg, S. L. 2004. Versatile and open software for comparing large genomes. Genome Biol. 5:R12.

Li, H. 2018. Minimap2: Pairwise alignment for nucleotide sequences. Bioinformatics 34:3094-3100.

Liao, M., Chen, C., and Li, Q. 2017. The complete mitochondrial genome of Alternaria alternata (Hypocreales: Nectriaceae). Mitochondrial DNA Part B 2: 587-588.

Marçais, G., and Kingsford, C. 2011. A fast, lock-free approach for efficient parallel counting of occurrences of k-mers. Bioinformatics 27:764-770.

Nguyen, H. D., Lewis, C. T., Lévesque, C. A., and Gräfenhan, T. 2016. Draft genome sequence of Alternaria alternata ATCC 34957. Genome Announce. 4:e0155415.

Pantou, M. P., Kouvelis, V. N., and Typas, M. A. 2008. The complete mitochondrial genome of Fusarium oxysporum: Insights into fungal mitochondrial evolution. Gene 419:7-15.
Stanke, M., Schöffmann, O., Morgenstern, B., and Waack, S. 2006. Gene prediction in eukaryotes with a generalized hidden Markov model that uses hints from external sources. BMC Bioinf. 7:62.

Tsuge, T., Harimoto, Y., Hanada, K., Akagi, Y., Kodama, M., Akimitsu, K., and Yamamoto, M. 2016. Evolution of pathogenicity controlled by small, dispensable chromosomes in Alternaria alternata pathogens. Physiol. Mol. Plant Pathol. 95:27-31.

Wang, M., Fu, H., Shen, X.-X., Ruan, R., Pun, N., Xu, J., Li, H., and Rokas, A. 2017. Genomic features and evolution of the conditionally dispensable chromosome in the tangerine pathotype of Alternaria alternata. bioRxiv Article 207746. doi:10.1101/207746

Wolters, P. J., Faino, L., van den Bosch, T. B. M., Evenhuis, B., Visser, R. G. F., Seidl, M. F., and Vleeshouwers, V. G. A. A. 2018. Gapless Genome Assembly of the Potato and Tomato Early Blight Pathogen Alternaria solani. Mol. Plant-Microbe Interact 31:692-694.

Woudenberg, J. H., Seidl, M. F., Groenewald, J. Z., de Vries, M., Stielow, J. B., Thomma, B. P., and Crous, P. W. 2015. Alternaria section Alternaria: Species, formae speciales or pathotypes? Stud. Mycol. 82:1-21. 